\title{
A confusing definition of disruption
}

\author{
Shijie $\mathbf{W u}^{1} \quad$ Qiang $W \mathbf{u}^{1 *}$
}

\begin{abstract}
It is important to measure whether a work is disruptive or developing. In 2016, Funk and Owen-Smith proposed novel indexes to evaluate the degree of a patent consolidating or destabilizing the technology streams (doi:10.1287/mnsc.2015.2366). Based on their methods, Wu et al. (2019) studied the disruption of articles, patents and software products, and made a conclusion that small teams are more disruptive than large teams (doi:10.1287/mnsc.2015.2366). Our research finds that the effect of $N_{R}$ on the disruption equation is contradictory as the sign of the disruption changes, and this problem may cause more trouble in any further work based on their indexes. When using other suitable approaches to measure how disruptive a work would be to the field of science or technology, we predict that there is no significant difference between large and small teams.
\end{abstract}

Keywords Innovation Measurement, Disruptive Work, Developing Work, Disruption, Citation Analysis, Research Evaluation, Metrics, Indicators

\section{Introduction}

It is important to measure whether a work is disruptive or developing. Wu et al. ${ }^{1}$ used an index that was previously designed by Funk and Owen-Smith ${ }^{2}$ to measure how disruptive a work would be to the field of science or technology. A flaw in this disruption measure raises concerns about the validity of their conclusions.

\section{The definition of disruption}

As Wu et al..$^{1}$ reported, Equation (1) shows the definition of the disruption:

$$
D=\frac{N_{F}-N_{B}}{N_{F}+N_{B}+N_{R}}
$$

*Corresponding author: Qiang Wu

E-mail: qiangwu@ustc.edu.cn

1 School of Management, University of Science and Technology of China, 96 Jinzhai Road, Hefei 230026, China. 
where $D$ is the disruption of the focal work, $N_{F}$ is the total number of works that cite just the focal work without citing any of the work's references, $N_{B}$ is the total number of works that cite both the focal work and any its references, and $N_{R}$ is the total number of works that cite just any of the focal work's references without citing the focal work itself (Fig. 1).

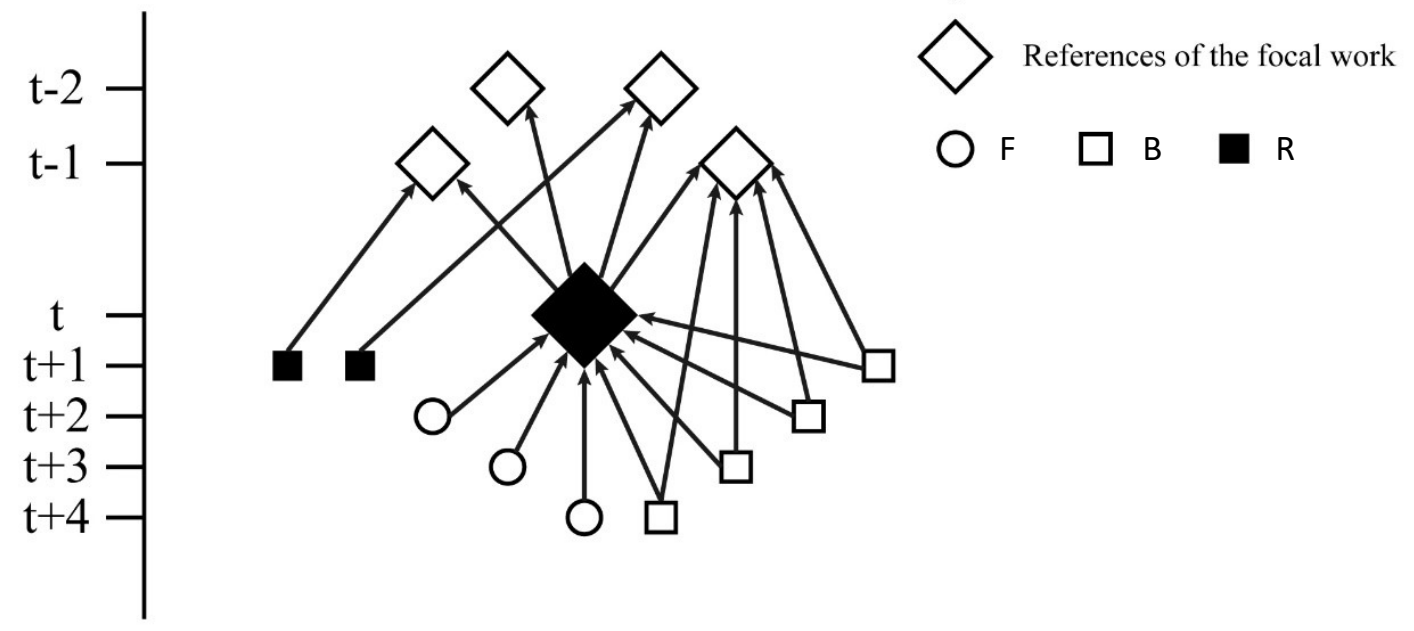

Fig. 1 Illustration of elements in citation networks

\section{Examples}

The first two lines of Fig. 1 indicate that a nonzero $N_{R}$ weakens the disruption of the focal work when the value of $\left(N_{F}-N_{B}\right)$ is positive. For example, by comparing works $\alpha$ and $\beta$ in Table 1, we can see that $\beta$ has a larger $N_{R}$ and ends up being judged less disruptive. This assessment fits the common sense that a larger $N_{R}$ should lead to a less disruptive work if the values of $N_{F}$ and $N_{B}$ are fixed.

Table 1 A demonstration of measuring disruption as defined by Equation (1)

\begin{tabular}{|c|c|c|c|c|}
\hline Focal work & $N_{F}$ & $N_{B}$ & $N_{R}$ & Disruption \\
\hline$\alpha$ & 90 & 10 & 0 & 0.80 \\
\hline$\beta$ & 90 & 10 & 100 & 0.40 \\
\hline$\gamma$ & 10 & 90 & 0 & -0.80 \\
\hline$\delta$ & 10 & 90 & 100 & -0.40 \\
\hline
\end{tabular}


Things become complicated when the value of $\left(N_{F}-N_{B}\right)$ is negative. By Equation (1), the existence of $N_{R}$ enhances the disruption of the focal work when the value of $\left(N_{F}-N_{B}\right)$ is negative. By comparing works $\gamma$ and $\delta$ in Table 1, we can see that, both $\beta$ and $\delta$ have a large $N_{R}$ value. Accordingly, we may expect $\delta$ to be less disruptive than $\gamma$, just like $\beta$ is less disruptive than $\alpha$. However, if we apply the disruption measure reported by Wu et al. ${ }^{1}$, we find that $\delta$ is more disruptive than $\gamma$, since $\delta$ has a larger disruption $(-0.40>-0.80)$.

Such a confusing result implies that the given disruption measure cannot reveal the true disruption of a scientific or technological work in some cases. Therefore, in Wu et al.'s work ${ }^{1}$, any conclusion based on regressions fitting the relation between team size and disruption may be unreliable. Moreover, views disagreeing with $\mathrm{Wu} e t a l$. have been reported by some previous research efforts which showed that individuals working alone are less likely to make breakthroughs and more likely to end up with ordinary outcomes ${ }^{3}$, while larger teams tend to achieve higher-impact and more destabilizing results ${ }^{2,4}$.

\section{Conclusion}

In conclusion, the effect of $N_{R}$ on the disruption is contradictory as the sign of the disruption changes, and this problem may cause more trouble in any further work based on Equation (1). The authors consider it as a flaw in this disruption measure, and strongly recommend $\mathrm{Wu}$ et al. to redesign this approach or come up with a reasonable explanation for the inconsistency. When using other suitable approaches to measure how disruptive a work would be to the field of science or technology, the authors predict that there is no significant difference between large and small teams.

Acknowledgements This work was supported in part by the National Natural Science Foundation of China (grant number 71874173) and the Academic Division of Mathematics and Physics of the Chinese Academy of Sciences (grant number 2018-M04-B-004).

\section{References}

1. Wu, L., Wang, D. \& Evans, J. A. Large teams develop and small teams disrupt science and technology. Nature 566, 378-382, doi:10.1038/s41586-019-0941-9 (2019).

2. Funk, R. J. \& Owen-Smith, J. A Dynamic Network Measure of Technological Change. Manage. Sci. 
63, 791-817, doi:10.1287/mnsc.2015.2366 (2017).

3. Singh, J. \& Fleming, L. Lone Inventors as Sources of Breakthroughs: Myth or Reality? Manage. Sci. 56, 41-56, doi:10.1287/mnsc.1090.1072 (2010).

4. Wuchty, S., Jones, B. F. \& Uzzi, B. The increasing dominance of teams in production of knowledge. Science 316, 1036-1039, doi:10.1126/science.1136099 (2007).

Competing interests The authors declare no competing interests. 